Emittance at the SuperHILAC and the Bevalac Transfer Line*

G. F. Krebs, J. G. Kalnins, M. S. Abinante, J. R. Alonso, B. Feinberg, K. Fowler, J. Guggemos, J. Staples, R. Thatcher, and E. Zajec

\author{
Accelerator and Fusion Research Division \\ Lawrence Berkeley Laboratory \\ 1 Cyclotron Road \\ Berkeley, CA 94720
}

March 1989

*This work was supported by the Director, Office of Energy Research, Office of High Energy and Nuclear Physics, Division of High Energy Physics, of the U.S. Department of Energy under Contract No. DE-AC03-76SF00098. 


\title{
EMITTANCE AT THE SUPERHLLAC AND THE BEVALAC TRANSFER LINE
}

\author{
G.F. Krebs, J.G. Kalnins, M.S. Abinante, J.R. Alonso, B. Feinberg, K. Fowler, J. Guggemos, \\ J. Sliaples. R. Thatcher and E. Zijec, \\ Lawrence Berkeley Laboratory. \\ University of California, \\ 1 Cyclotron Roud \\ Berkeley Califomia, 94720
}

\section{Introduction}

The SuperHILAC (SHILAC) accelerator serves as a heavy-ion injector to the Lawrence Berkeley Laboratory's Bevaluc via $260 \mathrm{~m}$ transpon line. Since the acceptance, and hence the final extracted beam intensity of the Bevalus is very sensiuve to the emitunce and dispersion of the beam at injection, a program was undertaken to accurately characterize these parameters under a variety of operaing condirions.

\section{The Accelerators}

The SHILAC accelerator is capable of accelerating ions from hydrogen through uranium. Ions exil the SHILAC with an energy of about $8.5 \mathrm{MeV} / \mathrm{A}$ and a momenium spread of $0.3 \% \leq$ $\Delta \mathrm{p} / \mathrm{p} \leq 1.2 \%$. After injection into the Bevalac, the ions can be accelerated up to an energy of $2100 \mathrm{MeV} / \mathrm{A}$ (for $\mathrm{q} / \mathrm{m}=1 / 2$ ). Typical intensities exiting the Beyalac range from $10^{7}$ for the heivier elements to over $10^{10}$ for the lighter elements.

\section{The Transfer Line}

The Bevalac transfer line, composed of some 12 dipole nugnets and 32 quadrupole magnets as well as beam profile montors, transports beam from the exit of the SuperHILAC to injection into the Bevalac. The beam envelopes of the transfer line for the design optics are shown in Figure 1, The horizontal envelope is shown on top, the vertical envelope on the bottom, with the dispersion shown as a dashed line. The optics in the transfer line are complicated by the fact that it has two horizontal bend sections (see Fig. 1 ), one vertical bend section, as well as a rotated bend section. The momentum spread of the beam is large enough to significantly affect the beam size, and so the design optics requires that these four bend sections be tuned to be achromatic.

Emituance slits were installed at the exit of the SHILAC and near the end of the transfer line as shown in Figure 1. The slit plate was made of $1 / 8$ in. tantalum with 0.014 in. slit widths. The two slits plates were separated by about $2 \mathrm{~m}$. With a Faraday cup installed behind the downstream slit to measure the beam intensity information, slit 1 scanned across the beam in $1 \mathrm{~mm}$ steps. Slit 2 was then repositioned, and the scanning procedure was repeated. This arrangement is shown schematically in Fig. 2 . Intensity and slit position information were recorded and fed into a computer program that calculated moments and emittance areas. One such result, given in Fig. 3, shows the 30\%,60\% and $90 \%$ beam area emittance ellipses for a ${ }^{20} \mathrm{Ne}$ beam measured at the sliss located at the exit region of the SHILAC.

This work supponed by the Director, Office of Energy Reseurch, Office of High Energy and Nuclear Physics, Nuclear Science Division, U.S. Deparment of Energy under Contract DEACU3-76SF00098

\section{Emittance Exchange}

A comparison was made between the horizontal and vertical emittances at the exit of the SHLAC. Shown in Fig. 4 is a scatter plot of the vertical emittance, $\varepsilon_{y}$, vs the horizontal emirtance, $E_{X}$, at the exit of the SHILAC for $30 \%, 60 \%$ and $90 \%$ beam areas. The measurements were made on a variety of beams : $\mathrm{H}, \mathrm{He}, \mathrm{C}, \mathrm{Ne}, \mathrm{Si}, \mathrm{Ar}, \mathrm{Ca}, \mathrm{Fe}, \mathrm{Ni}$ and $\mathrm{Nb}$. The results are scattered about $\left(\varepsilon_{\mathrm{y}} / \varepsilon_{\mathrm{x}}\right)_{\text {SHILAC }}=1$. However, for any paricular SHILAC tune this ratio was fairly uniformly distributed in the range 0.4 to 1.8. The variation in SHILAC emittance is often due to beam misalignment in the accelerator showing up in the measurements as clipping of the emittance ellipse.

The transfer line has a rotated magnet section that mixes the horizontal and vertical emittince planes. In this section magnets are rotated $8.82^{\circ}$ clockwise with respect to the incoming beam. At the end of the section the magnets are rotated $21.5^{\circ}$ in a counterclockwise direction. To negale the emittance mixing, the design optics requires that this section be tuned to be achromatic, and the emittances in the horizontal and vertical planes have the same magnitude and orientation at the entrance to the rotated section. In order to examine emittance exchange due to the rotated magnets, we calculated the ratio of the emittances at the Bevalac and SHLAC, $E$ (Bevalac) $/ \varepsilon$ (SHILAC) for both the horizontal and verical planes and compared these rapios to the ratio of vertical to horizontal emittances at the SHILAC exit, $\left(\varepsilon_{y} / \varepsilon_{x}\right)_{\text {SHILAC }}$ Figure 5 shows the distribution of this ratio in the vertical plane for several different values of $\left(\varepsilon_{y} / \varepsilon_{x}\right)_{\text {SHILAC }}$. The vertical dispersion measured at the Bevalac slits was small, $\eta \leq 0.25 \mathrm{~m}$ for all beams measured as expected from the design optics and does not contribute significantly to the vertical emittance. The measurements shown in Figure 5 follow the type of dependence we might expect if the emirtances are mixed. That is, if the vertical emittance is greater than the horizontal emittance at the exit of the SHILAC, $\left(\varepsilon_{y} / \varepsilon_{x}\right)_{\text {SHILAC }}>1$, then the ratio of vertical emittance at the Bevalac to the vertical emittance at the SHILAC should be less than 1. In fact, we see that when $\left(\varepsilon_{y} / \varepsilon_{x}\right)_{\text {SHILAC }}=1$, then

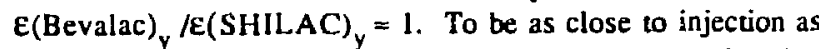
possible, required that the Bevalac slits be installed in a location where there is significant horizontal dispersion, $\eta_{x}=-2.29 \mathrm{~m}$ and the slope of the dispersion, $\eta_{x}^{\prime}=-0.045$. In Figure 6 we plot the ratio of the Bevalac to the SHILAC horizontal emittances against the SHILAC "mismatch" parameter, $\left(\varepsilon_{\mathrm{y}} / \varepsilon_{\mathrm{x}}\right)_{\text {SHILAC. }}$ The trend in the data is consistent with the emittance exchange in the verical plane. However, before we can look directly at the effect of the rotated section on the horizontal emittance, we first must deconvolute the dispersive part from the emittance measured at the entrance to the Bevalac. We cannot do this exactly, since we cannot measure all the necessary correlations between the $x, x^{\prime}$, and $\Delta p / p$ variables. Since the emittance ellipse near the Bevalac slits has only a small tilt (near a waist the Twiss functions $\alpha_{x}=0 \approx$ 
$\alpha_{y}$ ), then from beam optics for a Gaussian distribution ( for correlation coefficients $r_{12}=r_{16}=r_{26}=0$ ) we expect,

$$
\begin{aligned}
E_{0 \mathrm{x}} / \epsilon_{\mathrm{x}} & =\left(\mathrm{x}_{0} \mathrm{x}_{0}^{\prime}\right) /\left(\mathrm{x} \mathrm{x}^{\prime}\right) \\
& \left.=\sqrt{\left[1-\left\{\left(\eta_{\mathrm{x}} / \mathrm{x}\right) \Delta \mathrm{p} / \mathrm{p}\right\}^{2}\right]\left[1-\left\{\left(\eta_{\mathrm{x}}^{\prime} / \mathrm{x}^{\prime}\right) \Delta \mathrm{p} / \mathrm{p}\right\}^{2}\right.}\right]
\end{aligned}
$$

where $E_{\mathrm{x}}=$ the horizontal emituance

$\varepsilon_{0 x}=$ the dispersionless horizontal emittance

$x_{0}=$ the dispersionless horizontal beam size

$x_{0}{ }^{\prime}=$ the dispersionless horizontal beam divergence

$x=$ the horizontal beam size

$x^{\prime}=$ the horizontal divergence

$\Delta \mathrm{p} / \mathrm{p}=$ the beam momentum spread

$\eta_{\mathrm{x}}=$ the horizontal dispersion

$\eta_{x}^{\prime}=$ the slope of the dispersion

ln our study we measured $x, x^{*}$, and $\Delta p / p$. The dispersion, $\eta_{x}$, was measured at the Bevalac slit area, while $\eta_{x}$ was determined indirectly with the help of the design optics. Using these values and the above equation, we calculated the deconvoluted horizontal emittance, $\varepsilon_{0 x}$. The ratios of the deconivoluted Bevalac to SHILAC emittances in the horizontal plane, $\varepsilon$ (Bevalac) $x$ $/ \varepsilon(S H I L A C)_{x}$, versus the SHILAC emittance "mismatch" parameter, $\left(\varepsilon_{y} / \varepsilon_{x}\right)_{\text {SHILAC }}$ are shown in Figure 7 . The ratios $\varepsilon$ (Bevalac) $_{x} / \mathcal{E}$ (SHILAC) $)_{x}$ decrease as compared to the same ratio shown in Figure 6 and, as is the case of the vertical emittance shown in Figure 5 , there is no hoiizontal emittance exchange for $\left.\varepsilon_{\mathrm{y}} / \varepsilon_{\mathrm{X}}\right)_{\text {SHILAC }} \approx 1$.

\section{Conclusions}

The effects we have seen in characterizing the emittance in the Bevalac / SHILAC transfer line show a great deal of consistency. In the future we will improve the monitoring and diagnostics of the beam to ensure proper injection into the transfer line and may incorporate, if possible, an on-line emittance meusurement to help with tuning the accelerator. Measurements will continue in the future with the specific goal of understanding the $\eta$ dependence on the SHILAC tunes.

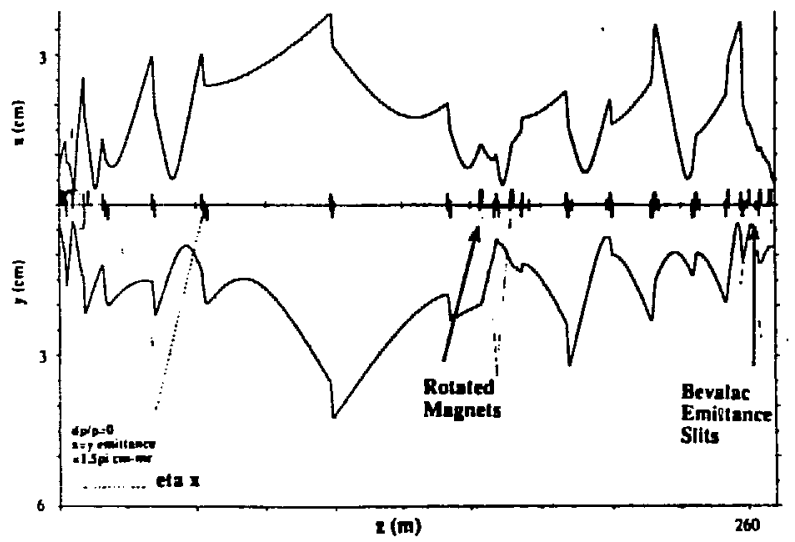

FIG. I BEVALAC TRANSFER LINE

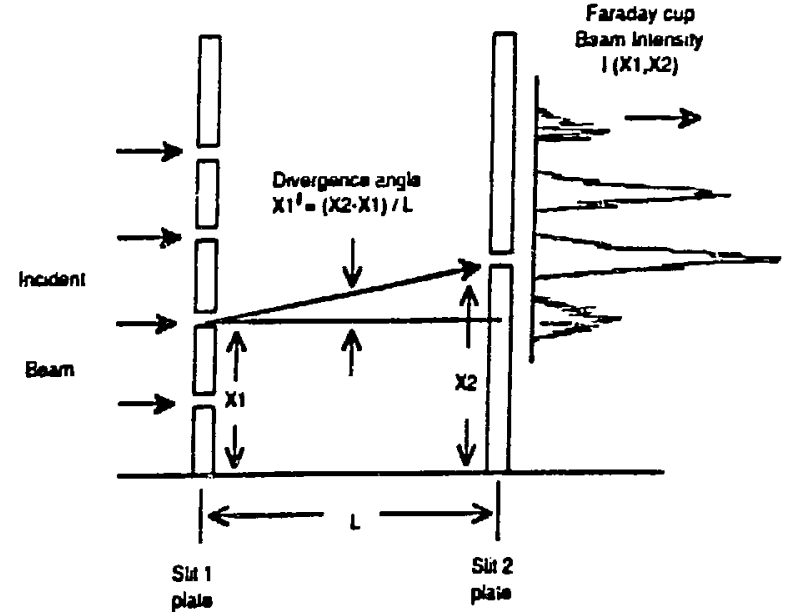

Fig. 2 Schematic of the
Emittance Slits

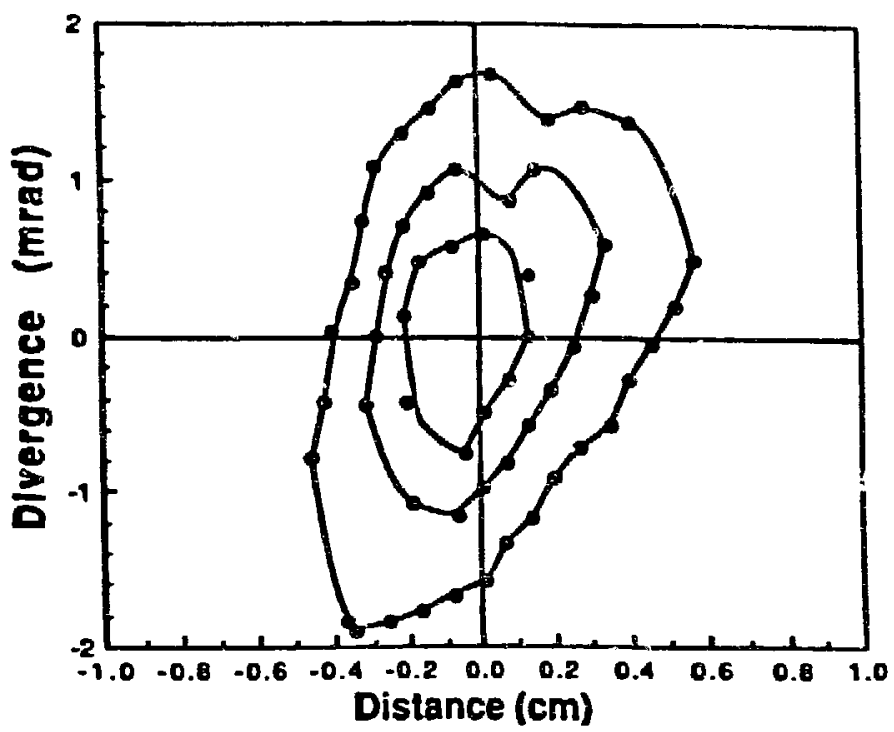

Fig. 3 Vertical Emittance Ellipses for $30 \%, 60 \%$ and $90 \%$ Beam Areas

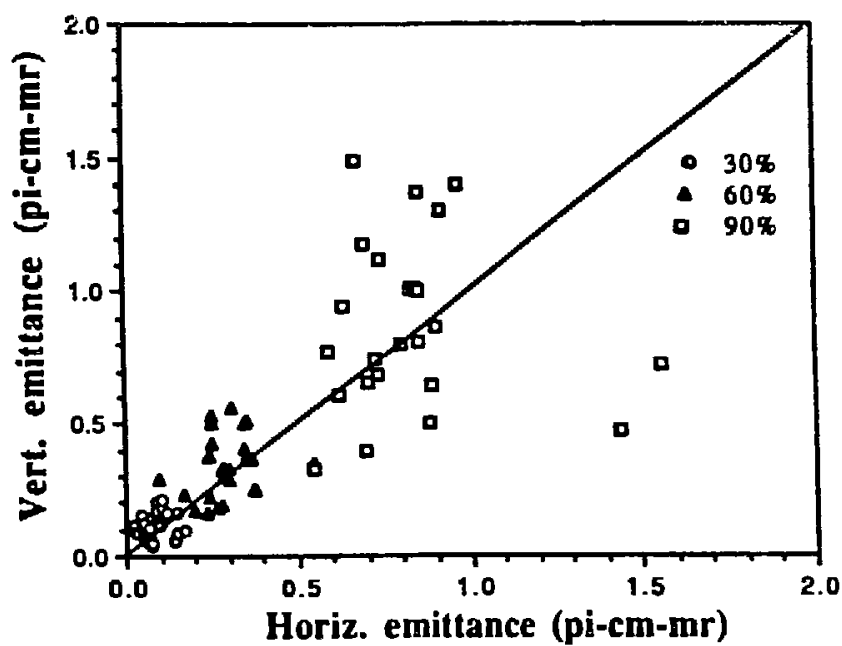

Fig. 4 The vertical vs Horizontal Emittances (30\%,60\%,90\% beam areas) 


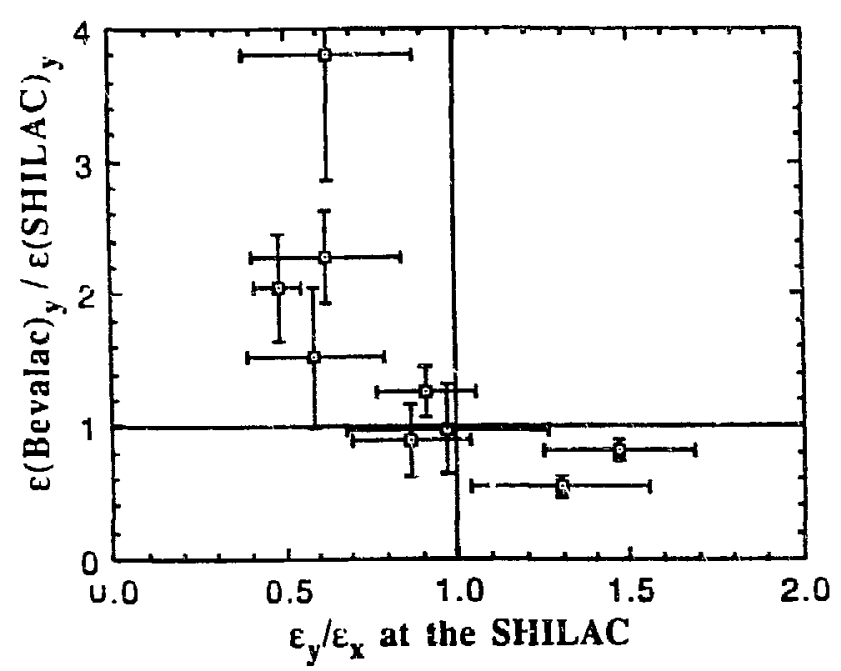

Fig. 5 The Ratio of the Bevalac to the SHILAC Vertical Emitiances vs the ratio of the Vertical to Horizontal SHIL AC emittances.

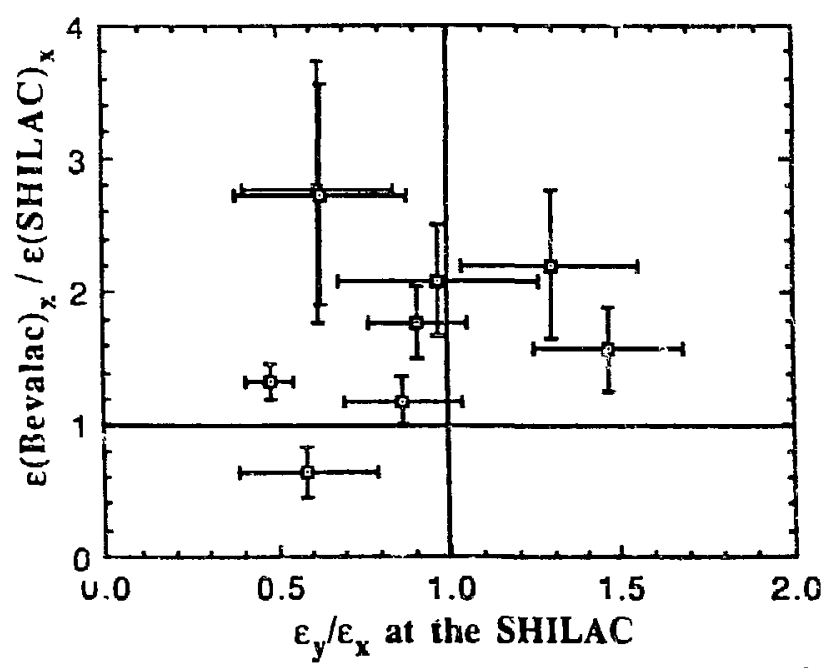

Fig. 6 The Ratio of the Bevalac to the SHILAC Horizontal Emittances vs the ratio of the Vertical to Horizontal SHILAC emittances.

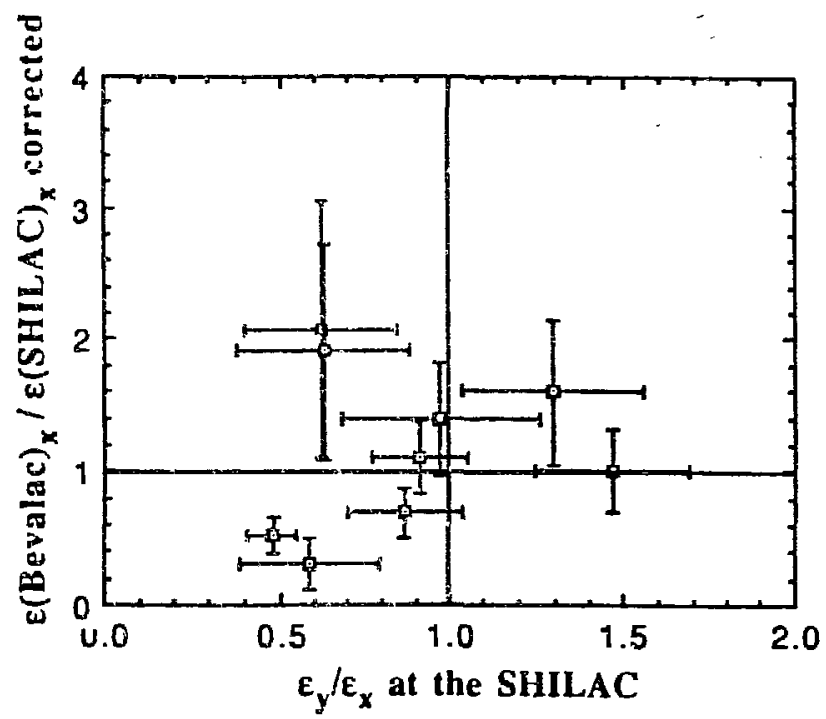

Fig. 7 The Corrected Ratio of the Bevalac to the SHILAC Horizontal Emittances vs the ratio of the Vertical to Horizontal SHILAC emittances. 